\title{
STUDIES IN THERMO-LUMINESCENCE.
}

II. Variations in the Decay of Phosphorescence in Balmain's Paint Produced by Heating.

By C. A. Pierce.

$\mathrm{I}^{\mathrm{N}}$ an earlier paper, ${ }^{1}$ on thermo-luminescence of Sidot blende, mention was made of the interesting character of corresponding curves for Balmain's paint. These curves were obtained with the apparatus used for the study of Sidot blende. A mercury lamp was used to excite the calcium sulphide, which was in powder form. Since the lamp was made of ordinary glass and the light from it was reflected at a mirror before reaching the powder, the excitation was due to the visible spectrum in the mercury arc. Before each excitation the powder was exposed to infra-red rays in an attempt to bring it to a standard condition.

The action of infra-red rays upon the phosphorescence of calcium sulphide is not so strong as their action upon the phosphorescence of Sidot blende. In the latter case, phosphorescence due to a very long excitation can be killed off almost immediately by infra-red rays and only the highest allowable temperature, just under dull red heat, is able to produce thermo-luminescence without renewed excitation. In the case of calcium sulphide a very long exposure to infra-red rays was necessary to kill off the phosphorescence, and no exposure was found to be long enough to suppress the thermo-luminescence completely. At the beginning of the experiments on calcium sulphide this fact was not recognized. If it had been, the powder could have been brought to an approximately standard condition by heating to a temperature a little higher than the highest temperature at which thermo-luminescence was to be studied. Fortunately, in every case the powder was exposed for one minute to infra-red rays of constant strength, hence it was always brought to a semi-standard condition. No extended attempt 
was made to compare the two methods, since the blue phosphorescence of calcium sulphide is a difficult color to measure in the photometer.

The fluorescence spectrum of this sample of calcium sulphide is shown in Fig. 23. This curve was obtained by comparison with the

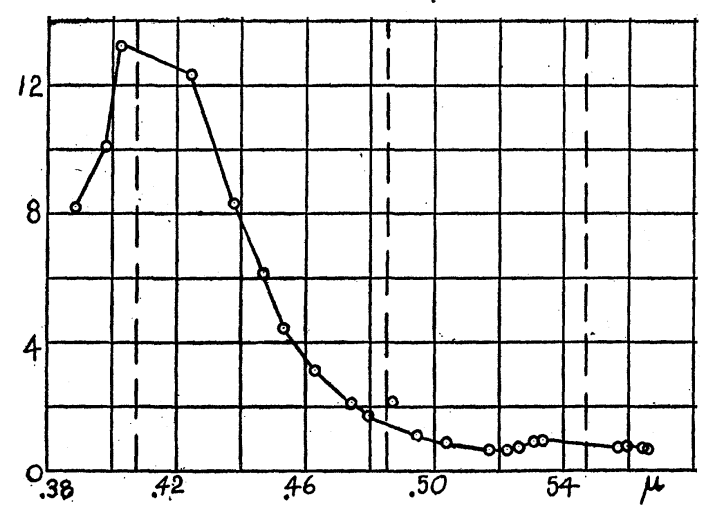

Fig. 23. Fluorescence spectrum of calcium sulphide excited by the visible spectrum of a mercury arc.

light reflected from the surface of a block of magnesium carbonate illuminated by an acetylene flame. Two bands are indicated by the curve, one with a maximum at about $0.4 \mathrm{I} \mu$ and the other with a maximum at about $0.54 \mu$. The dotted vertical lines show the wave-lengths of the lines of the mercury arc. While the figure indicates a continuous spectrum this is probably due to an error caused by stray light in the spectrophotometer. The effect of this error is twofold; to lower the zero line with respect to the curve and to unduly exaggerate the ratio of the maximum of the band at $0.4 \mathrm{I} \mu$ to the maximum of the band at $0.54 \mu$. Notwithstanding this correction, the band in the blue is much more intense than the other. This fact was shown by the apparent color of the initial phosphorescence, which was blue.

The effect upon the decay curve of varying the length of excitation is shown in Fig. 24. These curves are plotted with distances of the standard light from the photometer as ordinates and time measured from the end of excitation as abscissæ. For short excitations the curves are concave downward throughout, but for longer excitations 
the bending is concentrated in the first part of the curves. These curves are similar to those obtained with Sidot blende, except that the phosphorescence lasts much longer. Hence it is possible to get points relatively nearer the origin. This fact allows one to make a tentative deduction regarding initial intensity and length of excitation at room temperature. A study of Fig. 24 indicates strongly that the initial intensity is greater the longer the excitation, which is the impression obtained when getting the curves. Though it is impossible, with this apparatus, to get a measurement much nearer

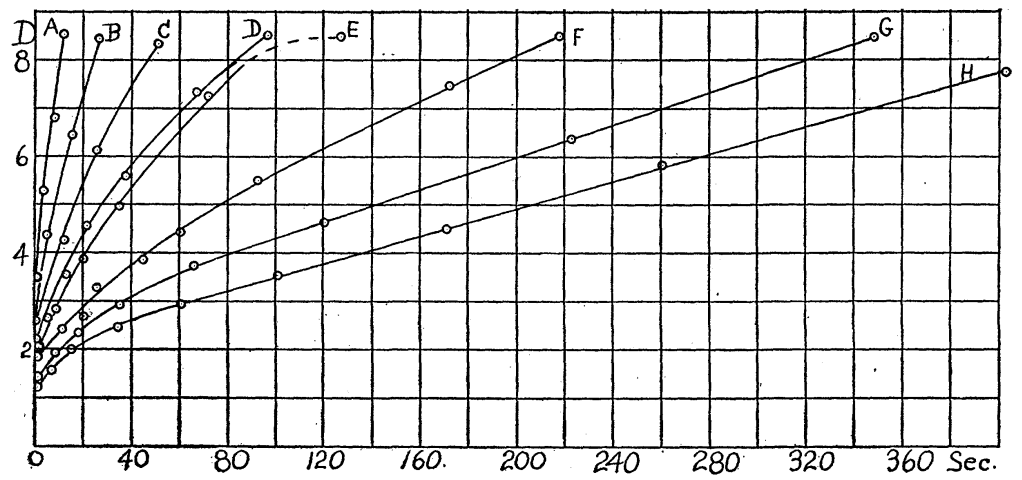

Fig. 24. Effect of varying the length of excitation. Excitation and decay at room temperature.

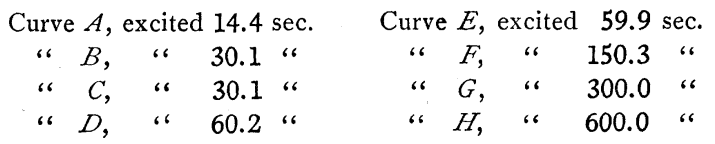

Curves $A, B, D$ and $F$ were taken on the second day and the remainder on the first day of the run.

the origin than 0.8 of a second, yet the eye is sensible to part of the change of intensity before this, giving one a means of estimating roughly the amount of change before the first measured point. The saturation effect is prominent as was the case with Sidot blende. Saturation is shown both by the change of initial intensity and by the change in slope of the curves as the excitation is increased.

In Fig. 24 some of the curves were obtained one day and the remainder on another day. One would expect curves $B$ and $C$ to coincide. The difference between them is probably due to the fact 
that the infra-red exposure does not reduce the powder to a standard condition. Curves $D$ and $E$ agree more closely than $B$ and $C$. A curve, not shown in the figure, excited for 300 seconds, coincides with $G$. This coincidence may have been due to chance, but it is what would be expected, because the effect of previous history becomes of less importance as the length of excitation is increased.

That the lack of agreement of curves $B$ and $C$ is due to the previous history of the powder is substantiated by the fact that curve $A$ was observed immediately before curve $B$, while curve $G$ was gotten immediately before curve $C$. No deductions can be made for curves $D$ and $E$ because the history of the powder previous to the excitation for curve $E$ is not known. Curve $D$ followed $B$ immediately, but some preliminary work without killing off was done before killing off for curve $E$.

The changes produced in the decay curve by varying the temperature at which excitation and decay take place are shown in

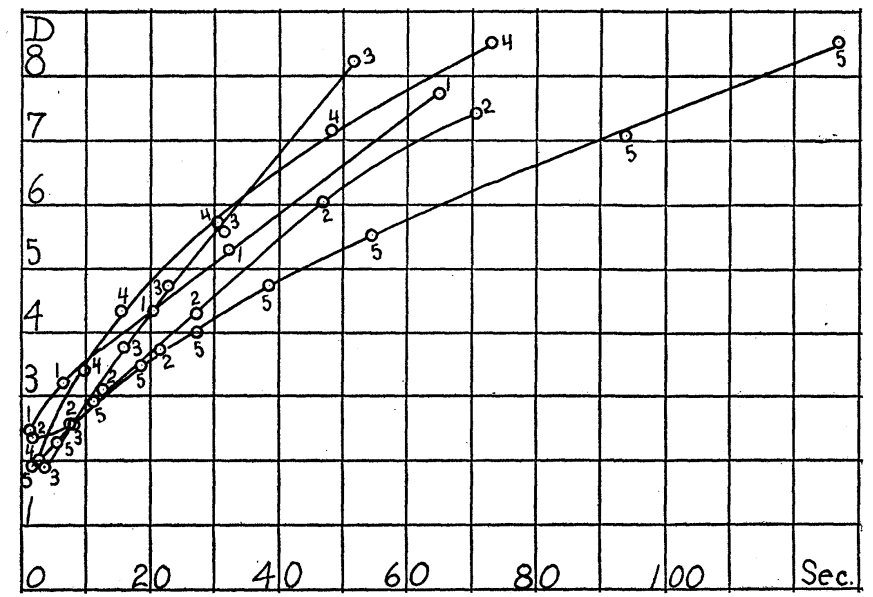

Fig. 25. Decay curves at different temperatures. Excited 2 minutes. Curve 1. Temp. of excitation and decay, room temp.

" 2 . “ " " "

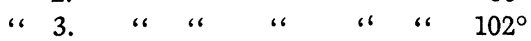

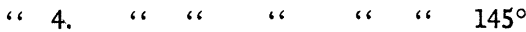

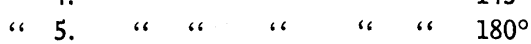

Fig. 25. The part that previous history plays in these curves cannot be estimated accurately, but its effect is small because the exci- 
tation, which is relatively a long one, was not changed during a set of curves. The curves were taken in the order that they are numbered. Curve I, taken at room temperature, is similar to the typical decay curves shown in Fig. 24. As the temperature is raised the curves pass through a series of changes. Curve 2 begins concave upward but changes during the decay to concave downward. Curve 3 is practically a straight line throughout. As the temperature is raised still further, the curves again become concave downward throughout, differing not widely from the typical decay curves at room temperature.

Another set of curves similar to those in Fig. 25 is shown in Fig.

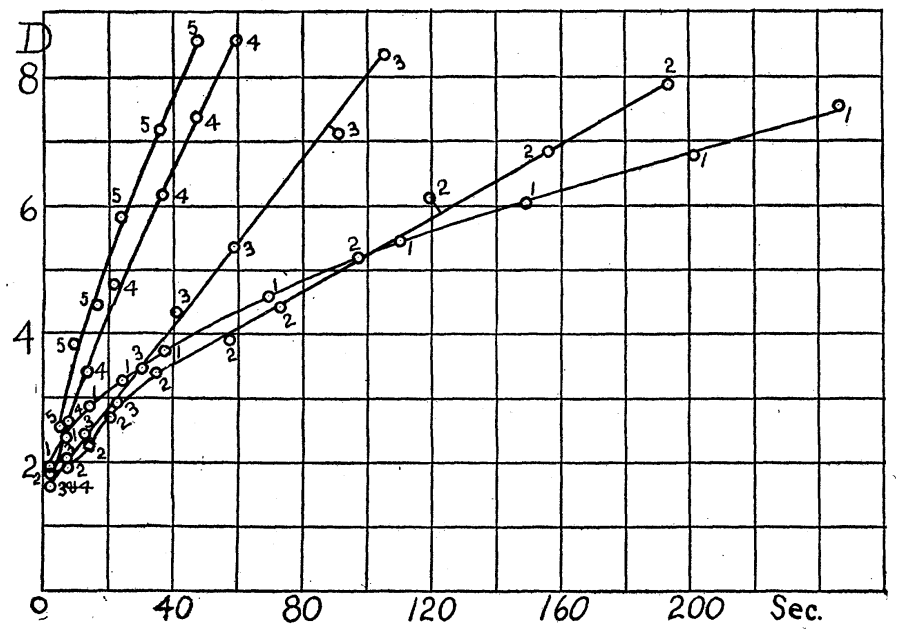

Fig. 26. Decay curves at different temperatures. Excited ro minutes. Curve 1. Temp. of excitation and decay, room temp.

\begin{tabular}{|c|c|c|c|c|c|c|}
\hline 2. & " & "، & “" & ، & "، & $42^{\circ} \mathrm{C}$ \\
\hline " 3 . & “" & “" & " & “" & " & $74^{\circ}$ \\
\hline " 4 . & ، & " & " & “ & " & $101^{\circ}$ \\
\hline “ 5. & " & ، & ، & ، & "، & $124^{\circ}$ \\
\hline
\end{tabular}

26, where the length of excitation is Io minutes. The double bending $^{1}$ is again exhibited in curve 2 .

${ }_{1}^{1}$ When taking the runs on which Fig. 25 is based, no importance was given to the single curve which shows double bending. Later, the runs on which Fig. 26 is based were made and the apparatus was rearranged for study of another substance before the significance of the double bending was recognized. On looking over all the curves taken on Balmain's paint, other indications of double bending were found, but none of them so pronounced as those shown above. It will be necessary to repeat the runs before the existence of the double bending can be satisfactorily established. 
The effect of previous history is shown in Figs. 27 and 28 . These curves might readily be mistaken for curves showing the

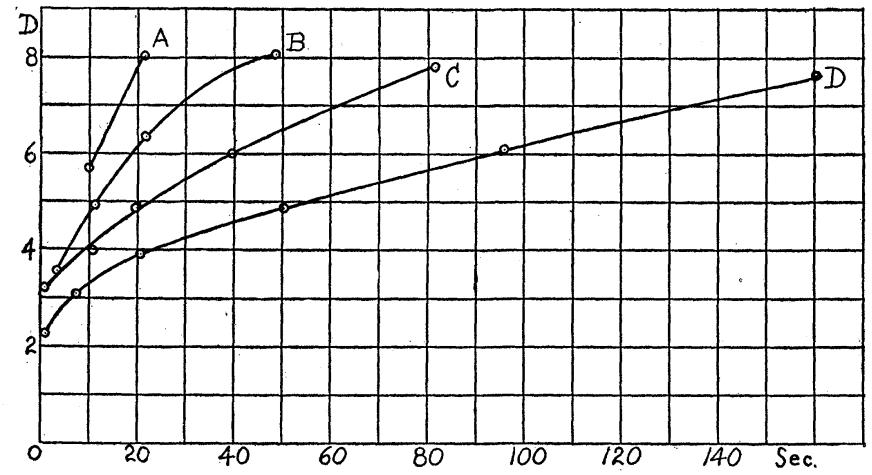

Fig. 27. Effect of history previous to excitation. Excitation and decay at room temperature. Excited 30 seconds. Curve $A$, excited after killing off with infra-red; curve $B$, excited immediately after curve $A$; curve $C$, excited immediately after curve $B$; curve $D$; before exciting for curve $D$, the powder was excited for 10 minutes and allowed to decay to an intensity corresponding to $D=9$ in the figure, at which time the excitation of 30 seconds was begun.

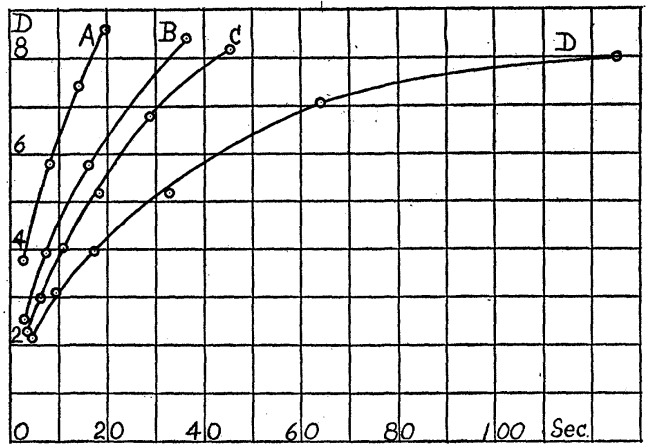

Fig. 28. Repeated curves in Fig. 27 at a temperature of $133^{\circ} \mathrm{C}$.

effect of varying the length of excitation. Fig. 29 also shows the effect of previous history combined, however, with the effect of temperature.

The decay of phosphorescence in the case of Balmain's paint was studied some years ago by F. J. Micheli, ${ }^{1}$ who has published decay curves of several phosphorescent substances, among them Balmain's paint.

\footnotetext{
${ }^{1}$ Arch. des Sci. Phys. et Nat., 19or, Vol. 12, p. 5.
} 
These curves were plotted with intensity $(I)$ and time as coördinates. For the sake of comparison, several of these curves have

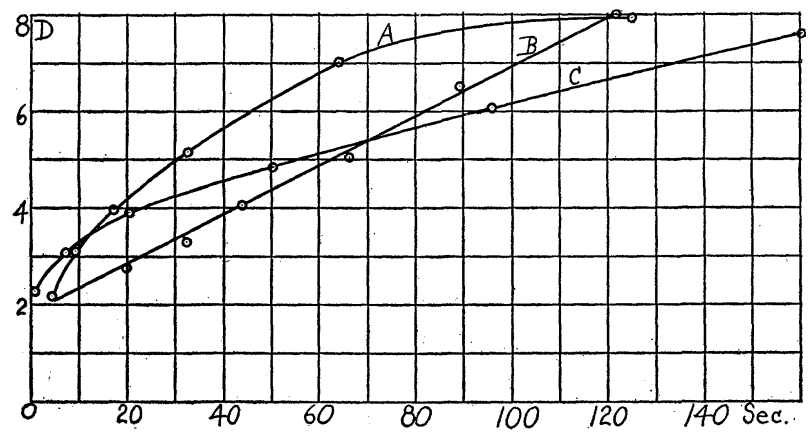

Fig. 29. Effect of history previous to excitation. Excited 30 seconds. Before each excitation, the powder was excited for Io minutes and allowed to decay to an intensity corresponding to $D=9$, at which time the excitation of 30 seconds was begun.

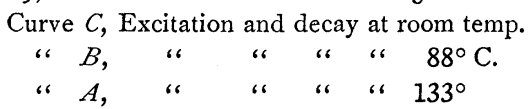

been replotted in Figs. 30 and $3 \mathrm{I}$ with $\mathrm{I} / \sqrt{I}$ and time as coördinates. Fig. 30 shows the effect of varying the length of excitation.

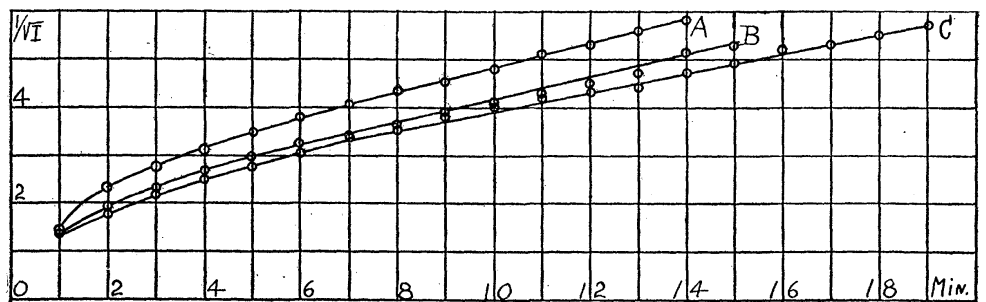

Fig. 30. Effect of varying the length of excitation. Excitation and decay at a tem. perature of $18^{\circ} \mathrm{C}$. (Replotted from the data of Micheli.)

$$
\begin{aligned}
& \text { Curve } A \text {, excited } 5 \mathrm{sec} \\
& \text { " } B, \text { " } 20 \text { " } \\
& \text { “ } C \text {, “ } 300 \text { ، }
\end{aligned}
$$

Ancther curve excited for 60 sec. coincided almost exactly with curve $C$.

Fig. 3 I shows the effect of varying the temperature at which excitation and decay take place. These curves and the curves given earlier in this article agree generally. Fig. 3 I shows the effect of excitation and decay at a temperature lower than any used in this article. 
In the case of calcium sulphide, it is not necessary to use a spectrophotometer to prove that there is more than one band included in the phenomenon of phosphorescence, for under the influence of

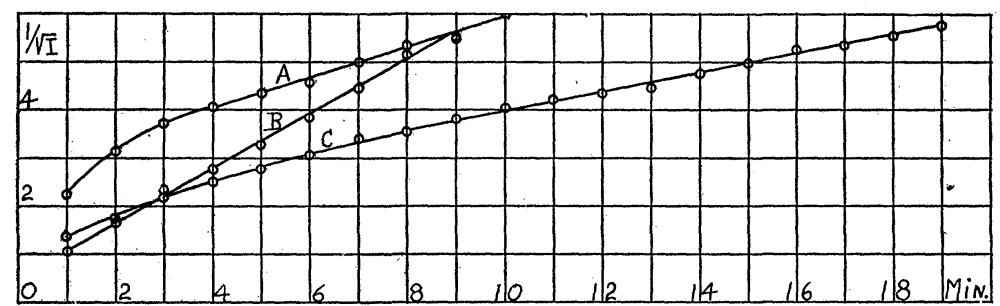

Fig. 31. Effect of varying the temperature at which excitation and decay takes place. Excited 300 seconds. (Replotted from the data of Micheli.)

$$
\begin{gathered}
\text { Curve } A \text {, temp. of excitation and decay, }-21^{\circ} \mathrm{C} \text {. } \\
\text { " } B \text {, “ } \\
\text { " }
\end{gathered}
$$

heat, the powder can be seen to change color, changing from a blue to a green. It has already been shown that the form of the phosphorescence decay curve may be closely approximated on the basis of two bands, each following the law,

$$
I=\frac{\mathrm{I}}{(a+b t)^{2}} .
$$

This explanation was carried to some length in the author's article on Sidot blende. But there seems to be no way of combining two decays each of which follows this law so as to produce an upward bending. If it be supposed that one band increases in intensity as the other decreases, then an upward bending is possible; but a downward bending is impossible unless both the bands subsequently decay together. This suggests that one of the bands is due to some secondary effect, instead of being produced by the exciting light. Such a band would increase in intensity a certain length of time, then decrease in intensity. If we suppose that the first band follows the law suggested, and that the second band follows the same law after it has reached its maximum intensity, then the discussion given in the article on Sidot blende still holds good, the supposition being that the second band is completely formed before the first point on a curve can be measured. If, however, conditions can be changed so 
that a point can be observed before the second band is completely formed, then the curve will show initially an upward bending, as in curve 2, Figs. 25 and 26. If a band is formed by some secondary effect it will usually be present in the fluorescence spectrum because the excitation is continued long enough for the band to be formed.

The effect of previous history can be explained without adding any complications to the ideas already set forth. In the case of Sidot blende, it was undoubtedly the band of longer wave-length that decayed the slower. The same is true, probably, for calcium sulphide, for the color changes from blue to green when the powder is heated. In the case of the decay curve at room temperature, the intensity is too small for the change in color to be recognized by the eye. Assuming that the band of longer wave-length decays more slowly, and that this band is due to some secondary effect, then the action of repeated excitations without intervening killing off by infra-red might be to increase the intensity and to decrease the rapidity of decay of the second band without introducing any change in the rapidity of decay of the first band. Some indications of these effects can be seen in Figs. 27 and 28.

It is unfortunate that the curves in Figs. 27 and 28 were not taken in the reverse order, i. e., excitation of long duration followed by shorter excitations. Such curves, according to the ideas just set forth, should show parallelism after the decay had proceeded some time. In an article on Sidot blende by Nichols and Merritt, ${ }^{1}$ several sets of curves were taken in this order and they exhibit parallelism. after decaying a short time.

To make any attempt to explain completely the curves in Figs. 25 and 26 on the basis of a second band due to secondary causes is impracticable because the problem is complicated by the addition of the effect of temperature. One assumption will necessarily have to be added, i. e., that there are certain temperatures at which either one or the other of the bands is most readily formed. This is a possible explanation of the fact that the decay curve becomes practically a straight line at one temperature.

A number of sets of curves were taken to show the effect of heating after decay has begun. For these curves the powder

' Phys. Rev., Vol. XXIII., No. I, July, 1906. 
was exposed to infra-red rays, then excited and allowed to decay at room temperature, heating being begun at a definite point on the decay curve. The coördinates for these curves are intensity $(I)$ and time. Figs. 32 and 33 show the effect of varying the length of excitation. The double flash mentioned in the case of Sidot blende is plainly evident in the curves, and in the experiment was evident to the unaided eye. The color also varied, being blue for the first flash and a yellowish green for the second. At the longest excitation, the second flash is barely visible in the photometer, the only

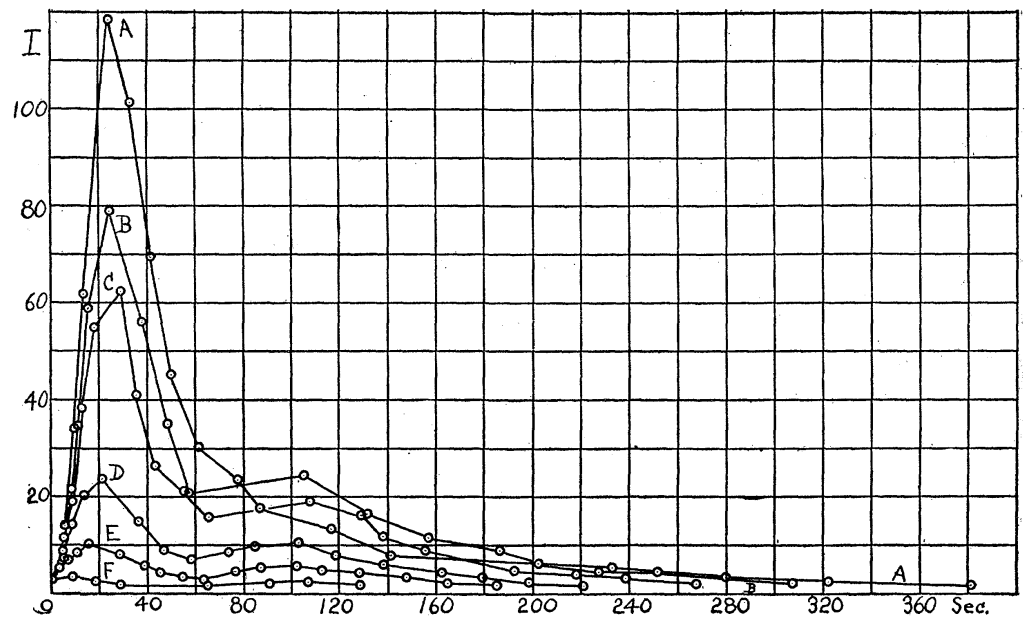

Fig. 32. Effect of varying the length of excitation. Excited and allowed to decay at room temperature to $I=2.9$, when heating was begun. Temperature of furnace, $277^{\circ} \mathrm{C}$.

$$
\begin{aligned}
& \text { Curve } A \text {, excited } 600.2 \text { sec. Curve } D \text {, excited } 60.7 \text { sec. } \\
& \text { " } B \text {, “ } 300.0 \text { " " } \quad \text { " } E \text {, “ } 30.1 \text { “ } \\
& \text { “ } C \text {, “ } 150.1 \text { “ } \quad \text { " } F, \text { “ } 14.8 \text { “ }
\end{aligned}
$$

evidence of its existence being a slowing up in the rate of decay. As the length of excitation is decreased, the second flash becomes, relative to the first, greater and greater.

At lower temperatures the second flash becomes less evident, and the peculiarity of the curve for 600 seconds excitation, as shown in Figs. 32 and 33, no longer exists. For the lower temperatures the second flash for 600 seconds excitation is greater than that for any shorter excitation at the same temperature. At a temperature near $100^{\circ} \mathrm{C}$., the second flash is no longer evident in the curves. 
It is evident that two bands are represented in the preceding curves. Any one of these curves is probably made up by the superposition of two curves, each of these component curves representing the

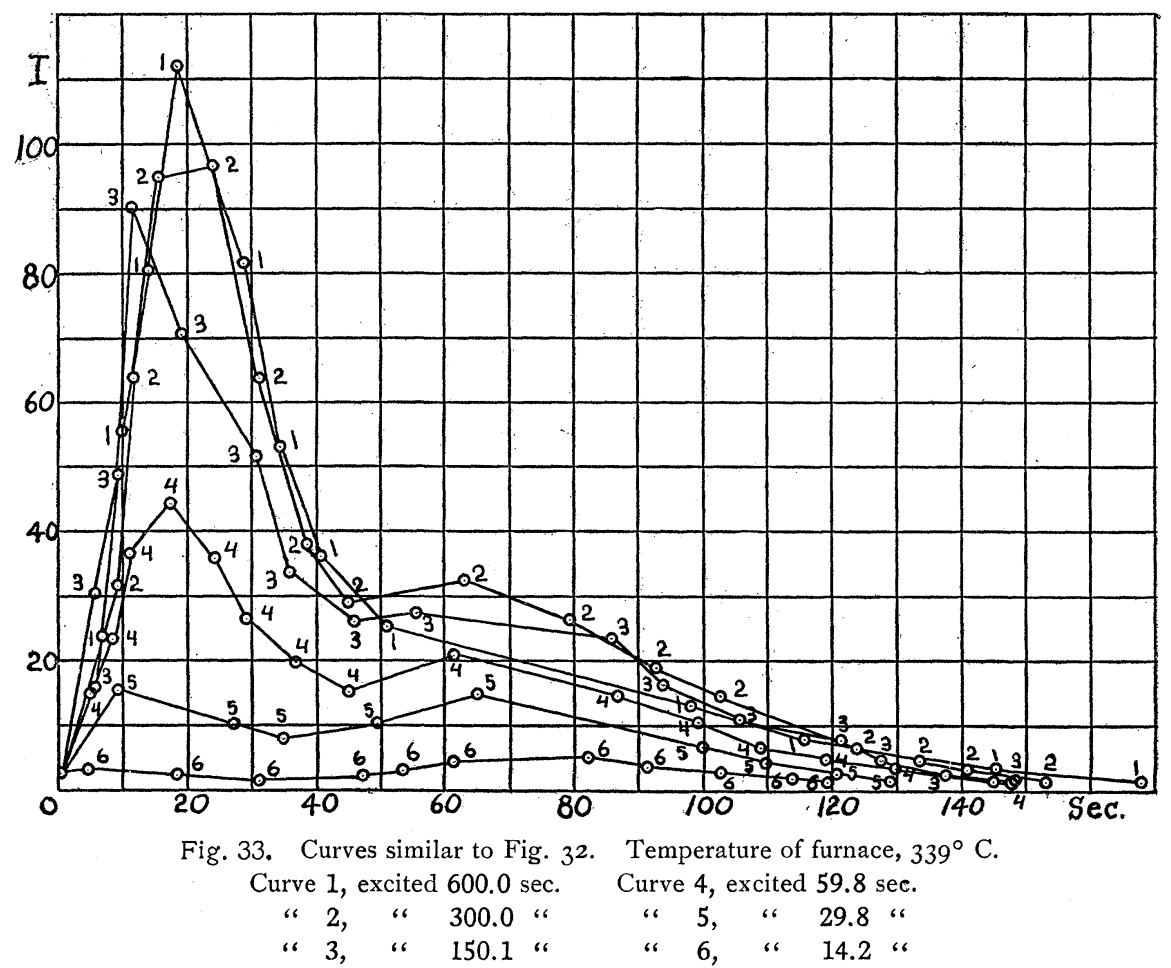

flash for one of the bands. If one considers the first flash in Figs. 32 and 33 , it will be seen that the curves resemble closely corresponding curves shown in the article on Sidot blende. The maximum intensity increases with length of excitation and occurs later and later. The areas included between the curves and the coördinate axes increase with the excitation. The effect of saturation is shown both by the change in areas and by the change in the maximum intensities. Fig. 34 shows the maximum intensities of the first flash plotted against length of excitation.

The curves in Figs. 32 and 33 and other figures not given here can be replotted to show the effect of changing the temperature of the furnace, when the length of excitation is constant. In this case, 
if one considers the first flash alone, the curves are similar to those already published for Sidot blende. Fig. 35 shows the maximum intensities of the first flash plotted against temperature.

The second flash does not always follow the laws of the first flash

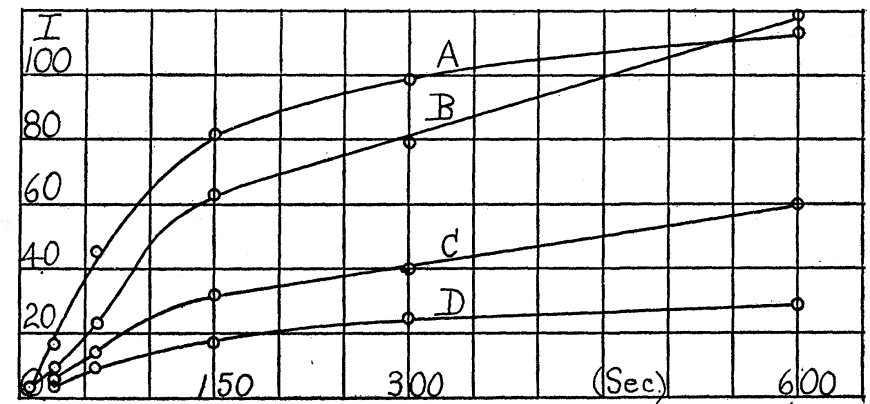

Fig. 34. Maximum intensities of the first flash plotted against length of excitation.

$$
\begin{aligned}
& \text { Curve 1, temp. of furnace } 339^{\circ} \mathrm{C} \text {. (From Fig. 33) } \\
& \text { “ } 2 \text {, “ “ “ } \\
& \text { " } 3 \text {, “ " “ } 4222^{\circ} \\
& \text { " } 4 \text {, “ " " } 4148^{\circ}
\end{aligned}
$$

as can be seen in Fig. 33. As the length of excitation is increased, the maximum intensity of the first flash occurs later and later, while the maximum of the second flash occurs earlier and earlier. This effect is not well defined in Fig. 32, due perhaps to the fact that the

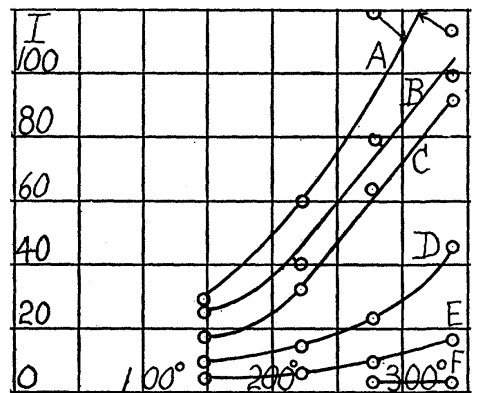

Fig. 35. Maximum intensities of the first flash plotted against temperature of the furnace.

$$
\begin{array}{cccrl}
\text { Curve } A \text {, excited } & 600 & \text { sec. } \\
\text { “ } & B, & \text { “ } & 300 & \text { “ } \\
\text { " } & C, & \text { “ } & 150 & \text { “ } \\
\text { “ } & D, & \text { “ } & 60 & \text { “ } \\
\text { " } & E, & \text { “ } & 30 & \text { “ } \\
\text { " } & F, & \text { " } & 15 & \text { “ }
\end{array}
$$


flash is of smaller intensity and consequently more difficult to follow accurately. Fig. 36 shows the effect of saturation for the second

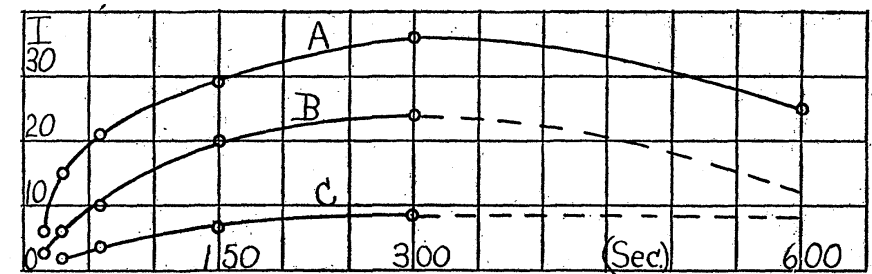

Fig. 36. Maximum intensity of the second flash plotted against length of excitation.

$$
\begin{aligned}
& \text { Curve } A \text {, temp. of furnace, } 339^{\circ} \text { C. (From Fig. 33) }
\end{aligned}
$$

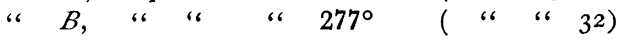

$$
\begin{aligned}
& \text { " } C \text {, “ " } \quad \text { " } 222
\end{aligned}
$$

flash. These curves indicate that an increase in the length of excitation beyond a certain length does not increase the maximum intensity but decreases it. There is a slight possibility that the change in maximum intensity noticed for an excitation of 600 sec-

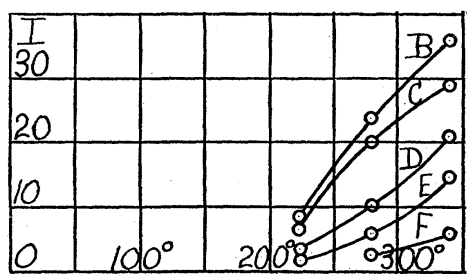

Fig. 37. Maximum intensity of the second flash plotted against temperature of the furnace.

$$
\begin{array}{ccccc}
\text { Curve } B \text {, excited } 300 & \text { sec. } \\
\text { " } & C, & \text { " } & 150 & \text { “ } \\
\text { " } & D, & \text { “ } & 60 & \text { “" } \\
\text { " } & E, & \text { “ } & 30 & \text { “ } \\
\text { " } & F, & \text { “ } & 15 & \text { “ }
\end{array}
$$

onds may be due to previous history, the excitation of 600 seconds always being the first run of a series. In contradiction to this is the fact that a practice run almost always preceded the series. Furthermore, the peculiar change in the second flash does not exist for lower temperatures, as curve C, Fig. 36, and other curves, indicate.

The relation between the maximum intensity of the second flash and the temperature is shown in Fig. 37. This figure corresponds 
closely to Fig. 35, which shows the corresponding relation for the first flash.

Fig. 38 shows the effect of delay in heating. Fig. 39 shows the same curves plotted with time measured from the beginning of

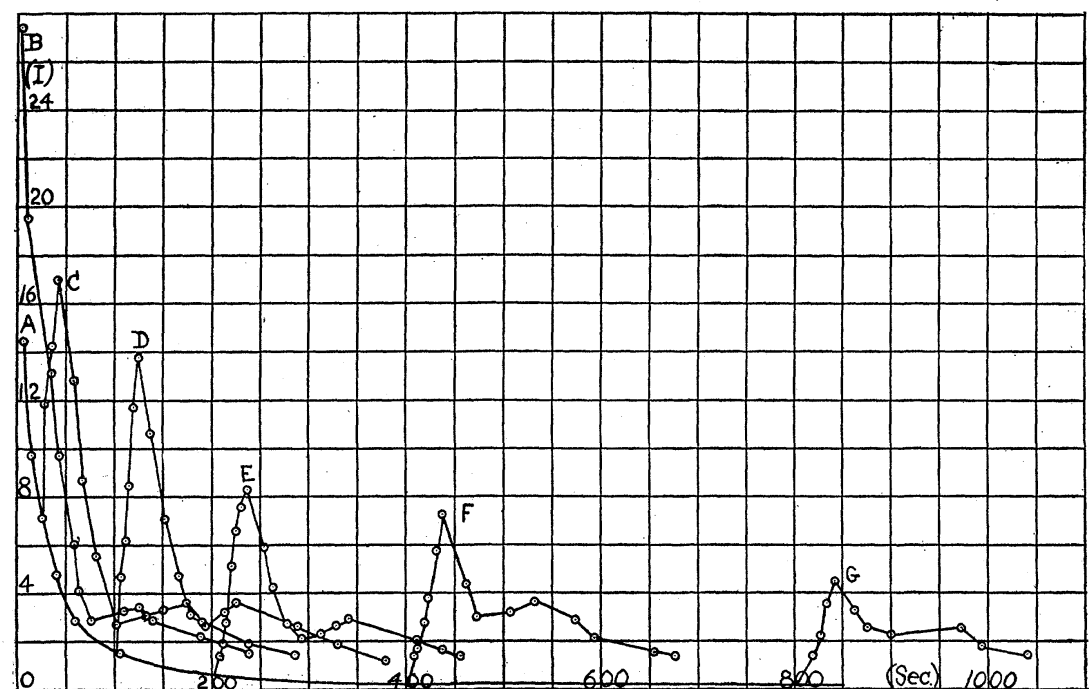

Fig. 38. Effect of delay in heating. 'Time measured from the end of excitation. Excited 60 seconds at room temperature. Temperature of furnace, $228^{\circ} \mathrm{C}$. Curve $A$, decay at room temperature. The time between the end of excitation and the beginning of heating is as follows :

$$
\begin{array}{cc}
\text { Curve } B, \quad 2.1 \text { sec. } \\
\text { “ } \quad C, 25.6 \text { “" } \\
\text { “ } \quad D, 101.7 \text { “ }
\end{array}
$$$$
\begin{array}{ccc}
\text { Curve } E, 203.2 \text { sec. } \\
\text { “ } & F, 401.7 \text { “ } \\
\text { “ } & G, 801.7 \text { “ }
\end{array}
$$

heating. Here again, if one considers the first flash alone, the curves are similar to those shown in the case of Sidot blende. The longer the delay in heating, the less intense the flash, and the later the maximum intensity of each flash. Furthermore, the time of decay for curves at one temperature and constant length of excitation is constant. It is difficult to make any deduction from the points representing the second flash.

Another set of curves similar to those shown in Fig. 38 is shown in Fig. 40. These curves show that the second flash becomes relatively larger with respect to the first as the excitation is shortened. Curve $C$ shows that the second flash, under suitable conditions, may become considerably larger than the first. 
It is difficult to say how much difference exists between the behavior of Sidot blende and Balmain's paint. The decay curves

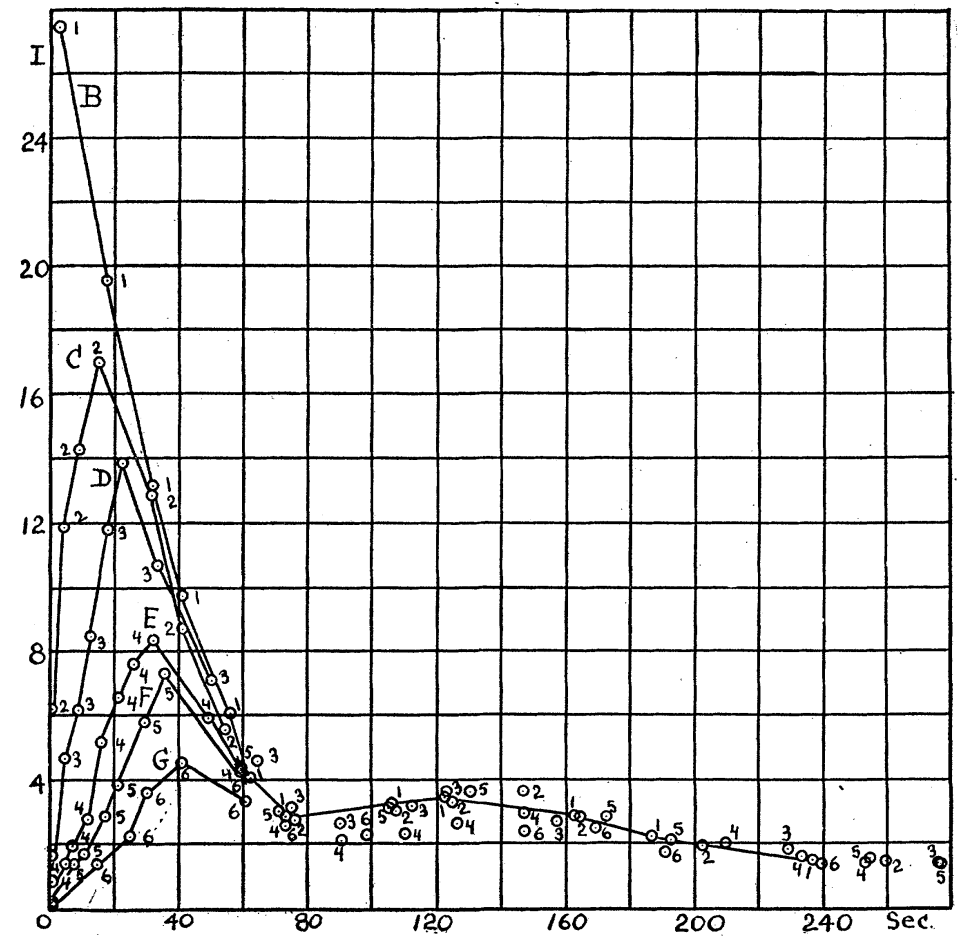

Fig. 39. Effect of delay in heating. Time measured from the béginning of heating. Same curves shown in Fig. $3^{8}$.

at room temperature are very much alike. As the temperature is raised both decay curves become straight lines, but Balmain's paint shows a transition through a double curvature decay before reaching the straight line decay, while Sidot blende does not exhibit this phenomenon. Above the temperature at which the decay curve becomes straight, Balmain's paint shows a decay approximating the decay at room temperature, while in the case of Sidot blende the decay is too rapid to be followed with the available apparatus. The decay of Sidot blende is so rapid that one cannot get enough points on a curve to say positively that it does not exhibit double curvature at temperatures much higher than room. In the cases where the powder is heated after excitation, Balmain's paint shows 
a double flash under some conditions, and one flash under other conditions, while Sidot blende shows one flash under all conditions,

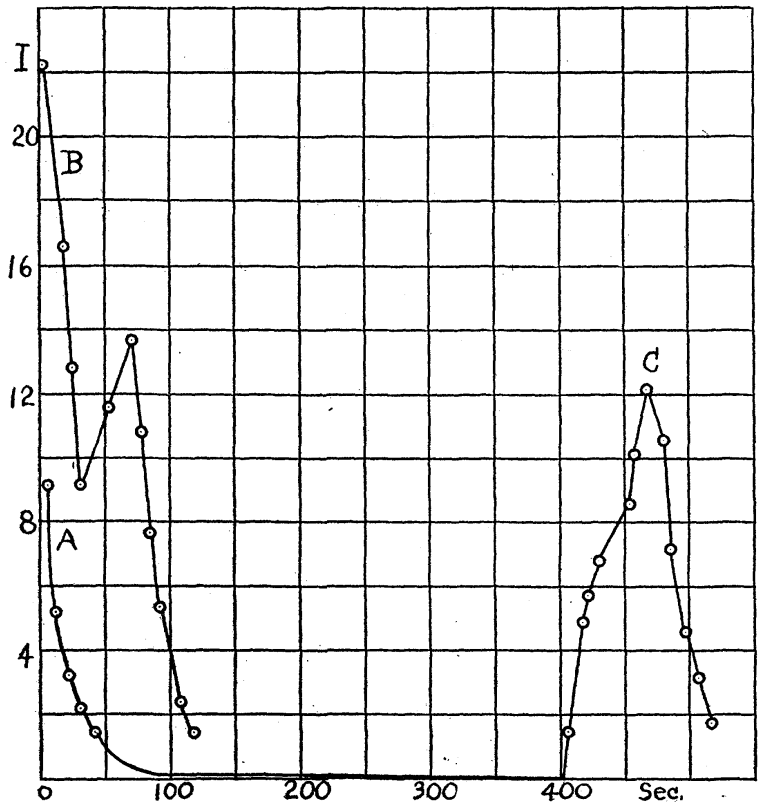

Fig. 40. Effect of delay in heating. Time measured from the end of excitation. Excited 30 seconds at room temperature. Temperature of furnace, $33^{\circ} \mathrm{C}$.

$$
\begin{aligned}
& \text { Curve } A \text {, decay at room temp. } \\
& \text { " } B \text {, waited } 2 \text { sec. after excitation before heating. } \\
& \text { " } C \text {, " } 400 \text { " " " }
\end{aligned}
$$

unless it be admitted that there are indications of a double flash in some of the Sidot blende curves, in which case the two substances show substantially the same curves. Considering the first flash only, there is perfect agreement between the curves of Sidot blende and Balmain's paint. The action of infra-red is more marked in the case of Sidot blende, but otherwise, there is no marked difference. The fluorescence spectrum shows two bands in the case of each substance, but there is one point of difference, in that the band of shorter wave-length is more prominent in the case of Balmain's paint and less prominent in the case of Sidot blende than the band of longer wave-length.

Physical Laboratory, CORNELL UNIVERSITY. 